\title{
ANTIPODAL EDGE-COLORINGS OF HYPERCUBES
}

\author{
Douglas B. West $^{1}$ \\ Zhejiang Normal University, Jinhua, China \\ and University of Illinois, Urbana, IL \\ e-mail: dwest@math.uiuc.edu \\ AND \\ JENNIFER I. WISE ${ }^{2}$ \\ Virginia Polytechnic Institute and State University \\ Blacksburg, VA \\ e-mail: jiwise@vt.edu
}

\begin{abstract}
Two vertices of the $k$-dimensional hypercube $Q_{k}$ are antipodal if they differ in every coordinate. Edges $u v$ and $x y$ are antipodal if $u$ is antipodal to $x$ and $v$ is antipodal to $y$. An antipodal edge-coloring of $Q_{k}$ is a 2edge-coloring such that antipodal edges always have different colors. Norine conjectured that for $k \geq 2$, in every antipodal edge-coloring of $Q_{k}$ some two antipodal vertices are connected by a monochromatic path. Feder and Subi proved this for $k \leq 5$. We prove it for $k \leq 6$.
\end{abstract}

Keywords: antipodal edge-coloring, hypercube, monochromatic geodesic.

2010 Mathematics Subject Classification: 05C55, 05C38.

\section{REFERENCES}

[1] M. DeVos and S. Norine, Edge-antipodal colorings of cubes, The Open Problem Garden.

http://garden.irmacs.sfu.ca/?q=op/edge_antipodal_colorings_of_cubes

[2] T. Feder and C. Subi, On hypercube labellings and antipodal monochromatic paths, Discrete Appl. Math. 161 (2013) 1421-1426. doi:10.1016/j.dam.2012.12.025

\footnotetext{
${ }^{1}$ Research supported in part by Recruitment Program of Foreign Experts, 1000 Talent Plan, State Administration of Foreign Experts Affairs, China.

${ }^{2}$ Research supported in part by NSF grant DMS 08-38434, "EMSW21-MCTP: Research Experience for Graduate Students".
} 
[3] K. Gandhi, Maximal monochromatic geodesics in an antipodal coloring of hypercube (2015), manuscript.

http://math.mit.edu/research/highschool/primes/materials/2014/Gandhi.pdf

[4] I. Leader and E. Long, Long geodesics in subgraphs of the cube, Discrete Math. 326 (2014) 29-33.

doi:10.1016/j.disc.2014.02.013

Received 23 January 2017

Revised 31 July 2017

Accepted 31 July 2017 